(C2017 IEEE. Personal use of this material is permitted. Permission from IEEE must be obtained for all other uses, in any current or future media, including reprinting/republishing this material for advertising or promotional purposes, creating new collective works, for resale or redistribution to servers or lists, or reuse of any copyrighted component of this work in other works. This is the author's version of an article that has been published in the conference proceedings. The final version of record is available at http://dx.doi.org/10.1109/VTCSpring.2017.8108335 


\title{
Waveform Parameter Selection for ITS Positioning
}

\author{
Michael Walter, Armin Dammann, Thomas Jost, Ronald Raulefs, Siwei Zhang \\ Institute of Communications and Navigation, German Aerospace Center (DLR) \\ Oberpfaffenhofen, 82234 Wessling, Germany \\ Email: \{M.Walter, Armin.Dammann, Thomas.Jost, Ronald.Raulefs, Siwei.Zhang $\} @$ DLR.de
}

\begin{abstract}
In this paper, we investigate the performance of mobile vehicle positioning based on signal propagation delay estimation in the uplink case for a realistic propagation environment. In order to optimize the ranging performance, we introduce a parametric waveform. This waveform contains a scalar parameter for adjusting the distribution of the available signal power over the frequency. The optimization is achieved by a functional dependency between the waveform parameter and the positioning error. In order to derive a cost function, we combine the approaches of the Cramér-Rao and Ziv-Zakai bounds for position and propagation delay estimation. As an exemplary environment we consider a mobile vehicle located in an area surrounded by three base stations together with realistic propagation conditions provided by the WINNER II channel model. The results show that the waveform parameter has to be adjusted differently compared to a simple free space propagation scenario. Additionally, we compare the obtained results with a scenario with four base stations and a scenario where we use the WINNER II channel model in terms of line-of-sight received power and shadow fading to classify the effects of geometry and propagation conditions.
\end{abstract}

\section{INTRODUCTION}

Nowadays, the parameters of future ITS communication systems for positioning are intensely discussed, e.g. in the European HIGHTS project [1]. At the moment infrastructurebased wireless communication technologies like Wi-Fi, ITSG5, UWB tracking, Zigbee, Bluetooth, LTE etc. are investigated in this project. The next major step in the development of mobile communication systems will be the 5G standard. Current research questions of $5 \mathrm{G}$ systems can be found in [2][4]. Besides requirements related to the communications part, the network-based positioning should be supported with an accuracy of $10 \mathrm{~m}$ down to less than $1 \mathrm{~m}$ in $80 \%$ of occasions and less than $1 \mathrm{~m}$ indoors according to [5]. Network-based positioning should should thus act as a complementary positioning system, if the position provided by a global navigation satellite system is not accurate enough. In the vehicular context this occurs for example in urban canyons [6]. Several system parameters envisaged for $5 \mathrm{G}$ in order to meet the challenging requirements related to communications are beneficial for positioning as well. These include higher carrier frequencies and signal bandwidths, dense networks and device-to-device communications, and the use of new waveform designs.

In this paper, we investigate how the shape of the waveform affects the positioning performance of the mobile vehicle (MV) depending on the channel conditions at a carrier frequency close to the ITS-G5 standard. In [7] the authors investigated combined Radar-communication waveforms, whereas we want to use multiple communication signals for one-way ranging directly for positioning. The parametric waveform can be adjusted by the MV to adapt it to its current needs of accuracy and latency (time-to-first-fix) in dependence on the expected signal-to-noise ratio (SNR). The ranging performance is evaluated in terms of the Cramér-Rao bound in conjunction with the Ziv-Zakai bound to determine its dependence on the waveform parameter $\gamma$ at different SNRs. We provide insights how the waveform will impact the position performance depending on the channel, the propagation conditions, and the number of base stations. The obtained results can help in defining a parametric waveform for future 5G mobile radio systems, since such a waveform integrates the demand of flexibility depending on the needs of the mobile vehicle by adapting to the propagation conditions.

The paper is structured as follows. The propagation environment is described in Section II. In Section III, we introduce the parametric waveform and in Section IV we define the system model and show how we evaluated the positioning performance. Section V presents the results and Section VI concludes the paper.

\section{Propagation Environment}

In order to describe the signal propagation between the $i$ th base station $\mathrm{BS}_{i}$ and a MV the typical urban macro cell channel model developed within the WINNER II project [8] is used similar to [9]. The path loss is given by the deterministic function

$$
\mathrm{PL}_{i}[\mathrm{~dB}]=A \log \left(d_{i}[\mathrm{~m}]\right)+B+C \log \left(\frac{f_{\mathrm{c}}[\mathrm{GHz}]}{5.0}\right) .
$$

It depends on the distance $d_{i}$ between $\mathrm{BS}_{i}$ and $\mathrm{MV}$ as well as the carrier frequency $f_{\mathrm{c}}$. The channel model parameters for a line-of-sight (LOS) or a non-LOS (NLOS) propagation scenario are summarized in Table I. The parameters have been calculated for typical heights of the $\mathrm{BS}_{i}\left(h_{\mathrm{BS}, i}=25 \mathrm{~m}\right)$ and the $\mathrm{MV}\left(h_{\mathrm{MV}}=1.5 \mathrm{~m}\right)$. In the WINNER II channel model for typical urban macro cell scenarios (C2), two sets of parameters are provided for (1) as shown in Table I for the LOS propagation case. The choice of the parameter set depends on the distance between receiver and transmitter $d_{i}$ and on the carrier wavelength $\lambda_{\mathrm{c}}=\frac{c_{0}}{f_{\mathrm{c}}}$ with $c_{0}$ being the speed of light. In our simulation scenario only the first parameter set, i.e., $d_{i}<48 \mathrm{~m}^{2} / \lambda_{\mathrm{c}} \approx 800 \mathrm{~m}$ for $f_{\mathrm{c}}=5 \mathrm{GHz}$, is relevant.

The WINNER II channel model distinguishes between LOS and NLOS propagation scenarios. The probability of a LOS scenario between $\mathrm{BS}_{i}$ and the MV

$$
P_{\mathrm{LOS} i}=\min \left\{\frac{18 \mathrm{~m}}{d_{i}}, 1\right\}\left(1-\mathrm{e}^{-\frac{d_{i}}{63}}\right)+\mathrm{e}^{-\frac{d_{i}}{63}}
$$




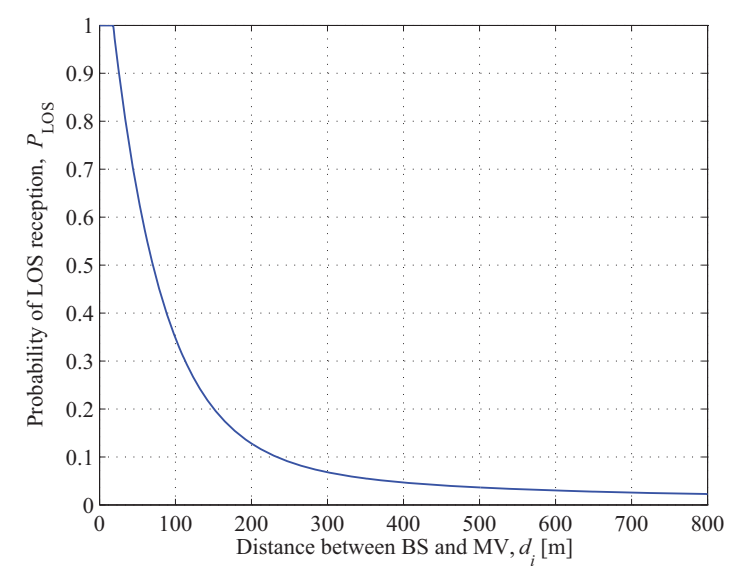

Figure 1. Probability for LOS condition between $\mathrm{BS}_{i}$ and the MV vs. their distance for the WINNER C2 Typical Urban Macro Cell channel model.

Table I

LARGE SCALE CHANNEL MODEL PARAMETERS FOR A TYPICAL URBAN MACRO CELL (WINNER C2).

\begin{tabular}{llcccc}
\hline $\begin{array}{l}\text { Propagation } \\
\text { Scenario }\end{array}$ & Range & $A$ & $B$ & $C$ & $\sigma_{\mathrm{SF}_{i}}$ \\
\hline LOS & $10 \mathrm{~m}<d_{i}<48 \mathrm{~m}^{2} / \lambda_{\mathrm{c}}$ & 26 & 39 & 20 & $4 \mathrm{~dB}$ \\
& $48 \mathrm{~m}^{2} / \lambda_{\mathrm{c}}<d_{i}<5 \mathrm{~km}$ & 40 & 12.4 & 6 & $6 \mathrm{~dB}$ \\
non-LOS & $50 \mathrm{~m}<d_{i}<5 \mathrm{~km}$ & 35.7 & 42.6 & 23 & $8 \mathrm{~dB}$ \\
\hline
\end{tabular}

depends on the distance $d_{i}$ between $\mathrm{BS}_{i}$ and the MV and is shown in Fig. 1.

The WINNER II channel model also includes shadow fading

$$
\mathrm{SF}_{i}[\mathrm{~dB}] \sim \mathcal{N}\left(0, \sigma_{\mathrm{SF}_{i}}\right),
$$

which is a random process and is drawn in $\mathrm{dB}$ from a normal distribution with zero mean and standard deviation $\sigma_{\mathrm{SF}_{i}}$. We assume that shadow fading is mutually uncorrelated between the links from the MV to the different BSs.

Path loss and shadow fading are related to the flat fading coefficients $\alpha_{i}^{\mathrm{BS}}$ as

$$
\alpha_{i}^{\mathrm{BS}}=10^{-\frac{\mathrm{PL}_{i}+\mathrm{SF}_{i}}{20}} .
$$

We are going to use these flat fading coefficients in Sec. IV.

\section{WAVEFORM DEFINITION AND ANALYSIS}

For propagation delay-based ranging, there is a trade-off between the estimation resolution and the detection ambiguities. For a given SNR, a dedicated power spectrum density (PSD) exists, which minimizes the mean-square error for range estimation. Subsequently, we introduce a waveform whose PSD is controlled by a scalar parameter. This waveform parameter is then used to optimize the range estimation performance for this type of waveform.

\section{A. Dirac-Rectangular Waveform}

We consider a parameterized band-limited waveform with bandwidth $B$. The waveform is built as a superposition of

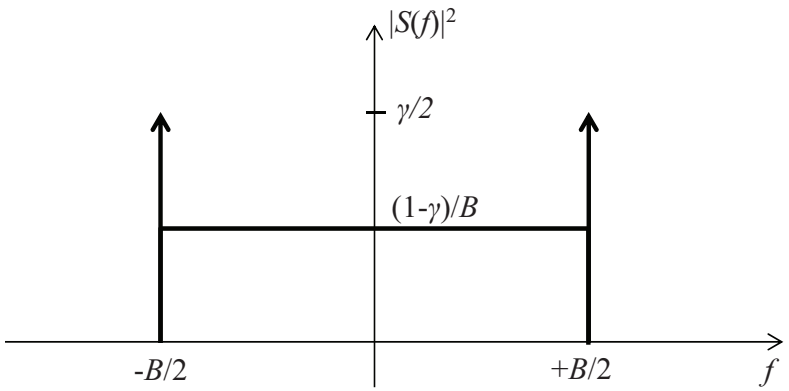

(a) Power spectrum density

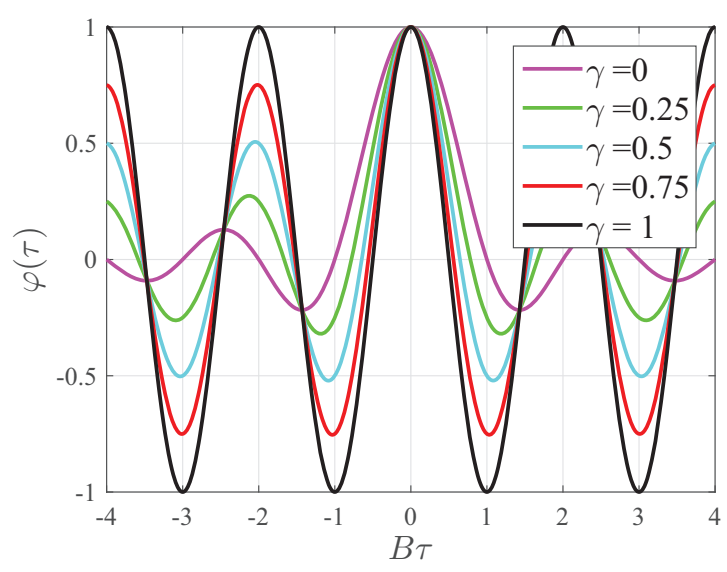

(b) Autocorrelation function

Figure 2. Dirac-rectangular waveforms

two component signals having Dirac and rectangular PSD, weighted by a scalar parameter $\gamma$. The resulting PSD is

$|S(f)|^{2}= \begin{cases}\frac{1-\gamma}{B}+\frac{\gamma}{2}\left[\delta\left(f+\frac{B}{2}\right)+\delta\left(f-\frac{B}{2}\right)\right], & |f| \leq \frac{B}{2} \\ 0, & |f|>\frac{B}{2},\end{cases}$

where $\delta(\cdot)$ stands for the Dirac distribution. The corresponding autocorrelation function, i.e. the Fourier transform of the PSD calculates to

$$
\varphi(\tau)=(1-\gamma) \frac{\sin (\pi B \tau)}{(\pi B \tau)}+\gamma \cos (\pi B \tau) .
$$

Fig. 2 shows both the PSD and autocorrelation function graphs for different values of the waveform parameter $\gamma \in[0,1]$. With an increasing $\gamma$, the signal power is more concentrated at the edges of the spectrum, which leads to a tighter mainlobe and higher sidelobes in the autocorrelation function.

\section{B. Range Estimation Performance Bounds}

The Cramér-Rao lower bound (CRB) is a lower bound for the achievable variance of any unbiased estimator. For signal propagation delay based range estimation between a transmitter and a receiver, the CRB according to [10] is

$$
\sigma_{\mathrm{CRB}}^{2}=\frac{c_{0}^{2}}{8 \pi^{2} \beta^{2} \frac{E_{\mathrm{s}}}{N_{0}}} .
$$

The CRB is inverse proportional to the squared equivalent signal bandwidth $\beta^{2}$ and the signal-to-noise ratio $\frac{E_{\mathrm{S}}}{N_{0}}$ experienced 


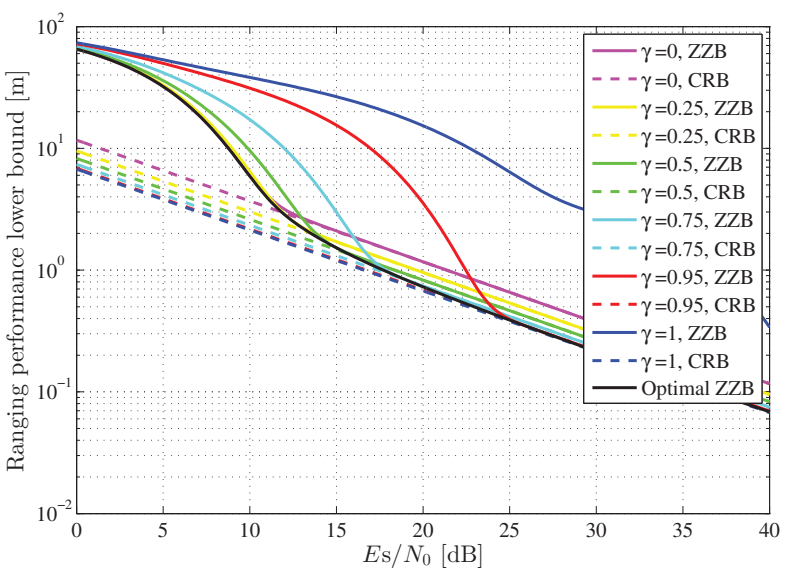

Figure 3. Square root of the ZZB and CRB for range estimation using Diracrectangular waveforms.

at the receiver. For the Dirac rectangular waveform the squared equivalent signal bandwidth is

$$
\beta^{2}=\frac{\int f^{2}|S(f)|^{2} \mathrm{~d} f}{\int|S(f)|^{2} \mathrm{~d} f}=\frac{B^{2}}{12}(1+2 \gamma), \quad 0 \leq \gamma \leq 1 .
$$

A larger $\gamma$ leads to a larger equivalent signal bandwidth, which reduces the ranging CRB. However, it comes with the price of higher autocorrelation function sidelobes as shown in Fig. 2(b). Particularly at low SNRs, an estimator might erroneously pick the delay of the sidelobe instead of the mainlobe with non-negligible probability. Due to this behavior, the estimation variance rapidly increases for lower SNRs. The $\mathrm{CRB}$, which is known to be tight only for reasonably high SNRs, does not take into account this threshold effect. The Ziv-Zakai lower bound (ZZB), however, considers this effect. We follow results in [11] for this type of bound. Accordingly, the ZZB for range estimation calculates to

$$
\sigma_{\text {ZZB }}^{2}=c_{0}^{2} \int_{0}^{T_{\text {obs }}} \tau\left(1-\frac{\tau}{T_{\text {obs }}}\right) \phi\left(\sqrt{\frac{E \mathrm{~s}}{N_{0}}(1-\varphi(\tau))}\right) \mathrm{d} \tau
$$

where

$$
\phi(x)=\frac{1}{\sqrt{2 \pi}} \int_{x}^{\infty} \mathrm{e}^{-t^{2} / 2} \mathrm{~d} t
$$

denotes the Gaussian Q-function. Parameter $T_{\text {obs }}$ describes the length of an observation interval, $E \mathrm{~s} / N_{0}$ the signal-tonoise ratio. The signal propagation delay, as the parameter to be estimated, is equally distributed within $\left[-T_{\text {obs }} / 2, T_{\text {obs }} / 2\right]$. Subsequently, we choose an observation interval length of $T_{\mathrm{obs}}=\frac{400 \mathrm{~m}}{c_{0}}=1.33 \mu \mathrm{s}$, which is aligned to a prior observation distance of $400 \mathrm{~m}$.

The square root of the $\mathrm{CRB}$ and the $\mathrm{ZZB}$ for range estimation are shown in Fig. 3 for a signal bandwidth of $B=10 \mathrm{MHz}$. The threshold effect mentioned above is clearly visible for the ZZBs. For increasing SNRs the ZZBs converge to the corresponding CRBs. The SNR values at which the ZZB converges to the CRB increases with increasing squared

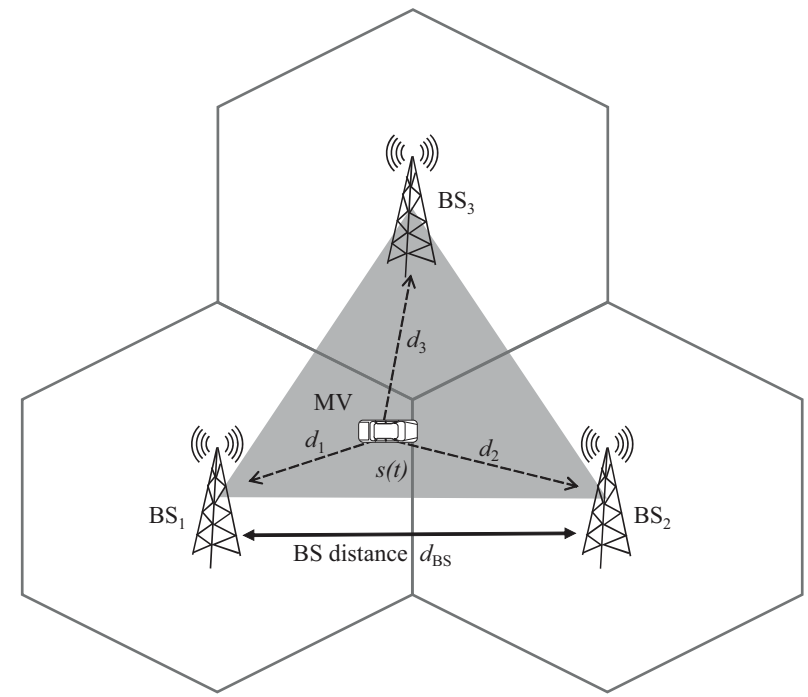

Figure 4. Mobile vehicle positioning using uplink signals.

equivalent bandwidth $\beta^{2}$. For the Dirac waveform, i.e. $\gamma=1$, the ZZB shows no convergence to the corresponding CRB. For this particular waveform, the autocorrelation function equals a cosine, so the amplitude of the sidelobes are equal to the mainlobe amplitude. Therefore, the autocorrelation function is ambiguous in its largest amplitude such that an estimation algorithm has equal probability in estimating the delay of a sidelobe instead of the mainlobe.

Minimizing the ZZB with respect to the waveform parameter $\gamma$ leads to an optimal ZZB which is also drawn in Fig. 3. This optimum is the lower envelope curve of the ZZB graphs for all $\gamma \in[0,1]$. Depending on the available signal power respectively the receiver SNR, we might decide for an optimum spectrum form. However, this depends on the local distribution of mobile vehicles. Therefore, it is beneficial to keep a positioning waveform flexible with respect to its power spectrum density.

\section{Positioning Performance Evaluation}

\section{A. Signal Model}

We consider an uplink transmission, where a MV transmits a signal $s(t)$ as shown in Fig. 4. At the base stations, the signals

$$
r_{i}(t)=\alpha_{i}^{\mathrm{BS}} s_{i}\left(t-\tau_{i}\right)+n_{i}(t), \quad i=1, \ldots, N_{\mathrm{BS}}
$$

are received. These received signals $r_{i}(t)$ consist of the delayed and attenuated transmit signal and additive white Gaussian noise (AWGN) $n_{i}(t)$. The observed propagation delays

$$
\tau_{i}(\boldsymbol{\theta})=\frac{d_{i}}{c_{0}}+T_{\mathrm{MV}}=\frac{1}{c_{0}} \sqrt{\left(x-x_{i}\right)^{2}+\left(y-y_{i}\right)^{2}}+T_{\mathrm{MV}}
$$

depend on the distance $d_{i}$ between the $\mathrm{MV}$ and $\mathrm{BS}_{i}$ and an unknown time offset $T_{\mathrm{MV}}$ between the MV and the BSs. The BSs itself are assumed to be synchronized. The unknown variables to be estimated, i.e., the position and time offset of the $\mathrm{MV}$, are gathered in a parameter vector $\boldsymbol{\theta}=\left[x, y, T_{\mathrm{MV}}\right]^{\mathrm{T}}$. 
We assume the propagation conditions as described in Sec. II. Therefore, the signal-to-noise ratios (SNRs)

$$
\mathrm{SNR}_{i}=\frac{E \mathrm{~s}_{i}}{N_{0}}=\frac{P \mathrm{~s}_{i}}{P_{\text {noise }}}=\frac{P_{\mathrm{Tx}} G_{\mathrm{Tx}} G_{\mathrm{Rx}, i} \alpha_{i}^{\mathrm{BS}}}{k_{\mathrm{B}} \vartheta B}
$$

observed at the BSs depend on the TX power $P_{\mathrm{Tx}}$, the antenna gains $G_{\mathrm{Tx}}$ and $G_{\mathrm{Rx}, i}$ at the $\mathrm{MV}$ and $\mathrm{BS}_{i}{ }^{1}$, and the flat fading coefficient $\alpha_{i}^{\mathrm{BS}}$ as introduced in (4). The noise power density $N_{0}=k_{\mathrm{B}} \vartheta$ is determined by the Boltzmann constant $k_{\mathrm{B}}$ and the system noise temperature $\vartheta$.

\section{B. Fisher Information for Positioning}

For the evaluation of the positioning performance of the $\mathrm{MV}$, we start with the calculation of the CRB for vector parameter estimations [10]. The unknown parameters which we wish to estimate are the position and time offset of the $\mathrm{MV}$, which we collect in a vector $\boldsymbol{\theta}=\left[x, y, T_{\mathrm{MV}}\right]^{\mathrm{T}}$. For the calculation of the CRB we require the so called Fisher information matrix. Its components

$$
\begin{array}{r}
\mathbf{F}_{k, \ell}=\mathrm{E}\left\{\left(\frac{\partial}{\partial \theta_{k}} \log \mathrm{p}(\mathbf{r}(t) \mid \boldsymbol{\theta})\right)\left(\frac{\partial}{\partial \theta_{\ell}} \log \mathrm{p}(\mathbf{r}(t) \mid \boldsymbol{\theta})\right)\right\} \\
=\frac{2}{N_{0}} \operatorname{Re} \int_{-\infty}^{+\infty} \sum_{i=1}^{N_{\mathrm{BS}}}\left(\frac{\partial}{\partial \theta_{k}} s_{i}\left(t-\tau_{i}(\boldsymbol{\theta})\right)\right) \\
\left(\frac{\partial}{\partial \theta_{\ell}} s_{i}^{*}\left(t-\tau_{i}(\boldsymbol{\theta})\right)\right) \mathrm{d} t
\end{array}
$$

are calculated from the likelihood function which in case of AWGN can be expressed as

$\mathrm{p}(\mathbf{r}(t) \mid \boldsymbol{\theta}) \propto \exp \left(-\frac{1}{N_{0}} \sum_{i=1}^{N_{\mathrm{BS}}} \int_{-\infty}^{+\infty}\left|s_{i}\left(t-\tau_{i}(\boldsymbol{\theta})\right)-r_{i}(t)\right|^{2} \mathrm{~d} t\right)$.

For notational convenience we omit constant factors, which vanish when calculating derivatives of the logarithmic likelihood function according to (14). The likelihood function provides the conditional probability density of observing the signals $\mathbf{r}(t)=\left[r_{1}(t), \ldots, r_{N_{\mathrm{BS}}}(t)\right]^{\mathrm{T}}$ at the $N_{\mathrm{BS}} \mathrm{BSs}$ for a given MV position and time offset, $\boldsymbol{\theta}=\left[x, y, T_{\mathrm{MV}}\right]^{\mathrm{T}}$. In matrix notation the Fisher information matrix

$$
\mathbf{F}=c_{0}^{2} \mathbf{J}_{\boldsymbol{\tau}}^{\mathrm{T}} \operatorname{diag}\left(\sigma_{\mathrm{CRB} 1}^{-2}, \ldots, \sigma_{\mathrm{CRB} N_{\mathrm{BS}}}^{-2}\right) \mathbf{J}_{\boldsymbol{\tau}}
$$

consists of the Jacobian matrix $\mathbf{J}_{\boldsymbol{\tau}}=\frac{\partial \boldsymbol{\tau}}{\partial \boldsymbol{\theta}}$ for the delay vector $\boldsymbol{\tau}=\left[\tau_{1}(\boldsymbol{\theta}), \tau_{2}(\boldsymbol{\theta}), \tau_{3}(\boldsymbol{\theta})\right]^{\mathrm{T}}$ and a diagonal matrix containing the inverse ranging variances, which are calculated from (7) for each link. Matrix $\mathbf{G}=c_{0} \mathbf{J}_{\boldsymbol{\tau}}$ is also called the geometry matrix. It only depends on the MV and BSs positions relative to each other. Note, the geometry matrix depends neither on the transmitted signal nor on the SNR. The dependency on the signal and propagation properties is solely contained in the diagonal ranging matrix $\operatorname{diag}\left(\sigma_{\mathrm{CRB} 1}^{-2}, \ldots, \sigma_{\mathrm{CRB} N_{\mathrm{BS}}}^{-2}\right)$. Finally, the CRB is calculated as the inverse of the Fisher information matrix. It contains lower bounds for the variance

\footnotetext{
${ }^{1}$ For simplicity, we assume that the antenna gains at the transmitter and receiver sites are equal for each link.
}

of an unbiased estimation of the unknown parameters - in our case $\boldsymbol{\theta}=\left[x, y, T_{\mathrm{MV}}\right]^{\mathrm{T}}$ - in its main diagonal.

\section{Waveform Optimization}

The CRB for ranging, as introduced in Sec. III-B and contained in (16), is known to be loose for low SNRs. This bound is monotonically decreasing with increasing squared equivalent bandwidth $\beta^{2}$, or equivalently, increasing parameter $\gamma$ for all SNRs. Minimizing the CRB with respect to the waveform parameter $\gamma$ results in an optimal waveform parameter $\gamma_{\mathrm{opt}}=1$, independent of the SNRs, and therefore, the MV position. The Ziv-Zakai lower bound (ZZB), however, does account for the threshold effect as shown in Fig. 3. It leads to an optimal choice of the waveform parameter $\gamma$ dependent on the SNR, or equivalently, the MV position.

As an approach for optimizing the positioning performance with respect to the waveform parameter $\gamma$ we replace the CRB ranging variances in (16) with the corresponding ZZB obtained from (9) and get

$$
\tilde{\mathbf{F}}=c_{0}^{2} \mathbf{J}_{\boldsymbol{\tau}}^{\mathrm{T}} \operatorname{diag}\left(\sigma_{\mathrm{ZZB} 1}^{-2}, \ldots, \sigma_{\mathrm{ZZB} N_{\mathrm{BS}}}^{-2}\right) \mathbf{J}_{\boldsymbol{\tau}}
$$

as a kind of modified Fisher information matrix. Its inverse

$$
\tilde{\mathbf{C}}=\tilde{\mathbf{F}}^{-1}
$$

provides $2^{\text {nd }}$ order moments for the estimation performance of the unknown parameters on its main diagonal. However, these values are formally no lower bounds but provide an easy to calculate cost function for waveform optimization. Similar to the CRB approach we use the square root

$$
\sigma_{\operatorname{pos}}(\gamma)=\sqrt{\tilde{\mathbf{C}}_{1,1}+\tilde{\mathbf{C}}_{2,2}}
$$

of the sum of the first two main diagonal elements of matrix $\tilde{\mathbf{C}}$ as a measure for the expectable position estimation standard deviation. This metric depends on the ZZB ranging variances $\sigma_{\mathrm{ZZB} i}^{2}$. Since the optimal choice of the waveform parameter $\gamma$ is dependent on the SNR, we expect an optimum

$$
\gamma_{\mathrm{opt}}=\underset{0 \leq \gamma \leq 1}{\arg \min } \sigma_{\mathrm{pos}}(\gamma)
$$

leading to an optimal (minimum) positioning error

$$
\sigma_{\mathrm{opt}}=\sigma_{\mathrm{pos}}\left(\gamma_{\mathrm{opt}}\right)
$$

according to (19). This optimal positioning error depends on the position of the MV as well as on further system parameters like the base station distance, effective power, etc.

\section{Results}

In this section, we investigate the influence of the WINNER II channel on the position estimation performance. The simulations are conducted with the parameters provided in Table II. An uplink scenario similar to the one shown in Fig. 4 is used, with one MV and three BSs.

The positioning performance is evaluated for $\gamma=0$ and $\gamma_{\text {opt }}$. Hereby, the case with $\gamma=0$, i.e. a waveform with a rectangular spectrum, is used as a reference, since typical today reference signals use a rectangular PSD. 
Table II

SIMULATION PARAMETERS.

\begin{tabular}{lll}
\hline Parameter & & Value \\
\hline Carrier frequency & $f_{\mathrm{c}}$ & $5 \mathrm{GHz}$ \\
Effective power & $P_{\mathrm{Tx}} G_{\mathrm{Tx}} G_{\mathrm{Rx}, i}$ & $10 \mathrm{dBm}$ \\
Signal bandwidth & $B$ & $10 \mathrm{MHz}$ \\
Boltzmann constant & $k_{\mathrm{B}}$ & $1.381 \cdot 10^{-23} \mathrm{Ws} / \mathrm{K}$ \\
Noise temperature & $\vartheta$ & $300 \mathrm{~K}$ \\
Noise power density & $N_{0}=k_{\mathrm{B}} \vartheta$ & $N_{0}=-173.8 \mathrm{dBm} / \mathrm{Hz}$ \\
Base station distance & $d_{\mathrm{BS}}$ & $50 \mathrm{~m}$ \\
\hline
\end{tabular}

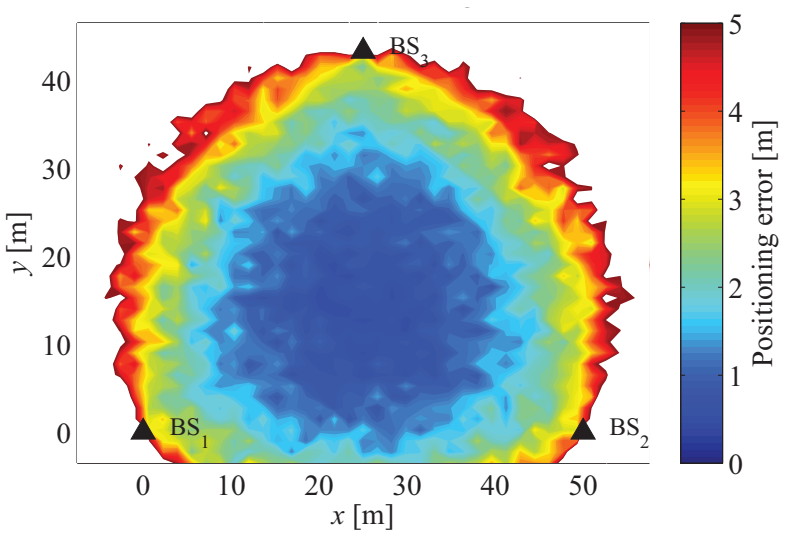

Figure 5. Positioning error $\sigma_{\text {pos }}(0)$ for a waveform with rectangular PSD $(\gamma=0)$.

For every position of the MV the received $\mathrm{SNR}_{i}$ at the $i$-th BS according to (13) is calculated. By inserting the resulting SNR into (9), we obtain the Ziv-Zakai bound for ranging estimation. The ZZB is then inserted into (17) to obtain the position error in dependence on the waveform parameter $\gamma$ according to (19). Since the path loss and the shadow fading are modeled by a stochastic process, it is not guaranteed to have three good SNR values, if the MV is close to the centroid of the triangle.

The positioning error for the reference case with $\gamma=0$ is shown in Fig. 5. Compared to a scenario, where only the free space loss is accounted for, the positioning error in Fig. 5 does not exhibit symmetry anymore. The stochastic shadow fading clearly prevents this. Since the probability of having LOS propagation is smaller than 0.5 for $d_{i}>70 \mathrm{~m}$, we restricted the scenario size to $d_{\mathrm{BS}}=50 \mathrm{~m}$. As the probability of one bad link to the BS increases, the position error naturally becomes larger.

The situation is similar for the positioning error using $\gamma_{\mathrm{opt}}$ as shown in Fig. 7. The slightly different color, however, indicates that the positioning error is smaller for the optimal waveform as expected from theory. We present the gain

$$
G=\frac{\sigma_{\mathrm{pos}}(\gamma=0)-\sigma_{\mathrm{opt}}}{\sigma_{\mathrm{pos}}(\gamma=0)}=1-\frac{\sigma_{\mathrm{opt}}}{\sigma_{\mathrm{pos}}(\gamma=0)}
$$

in dependence on the location of the MV in Fig. 6. The gain

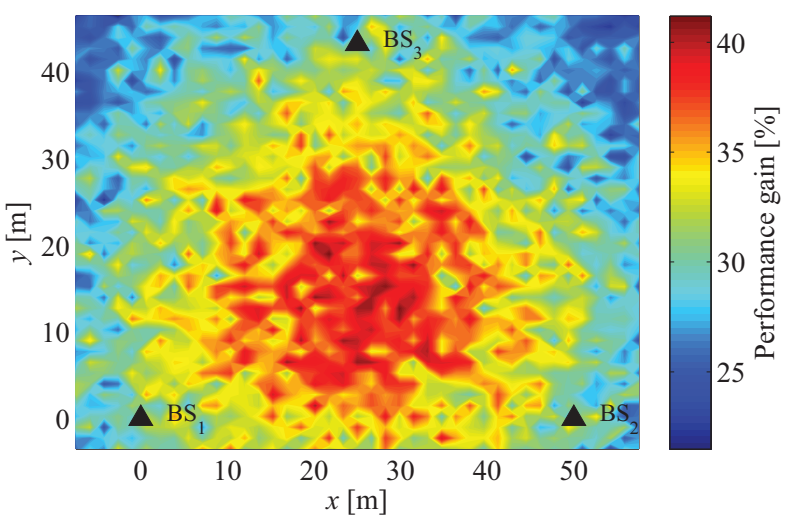

Figure 6. Performance gain $1-\sigma_{\mathrm{opt}} / \sigma_{\mathrm{pos}}(0)$ for the optimal waveform choice compared to a reference waveform with rectangular PSD.

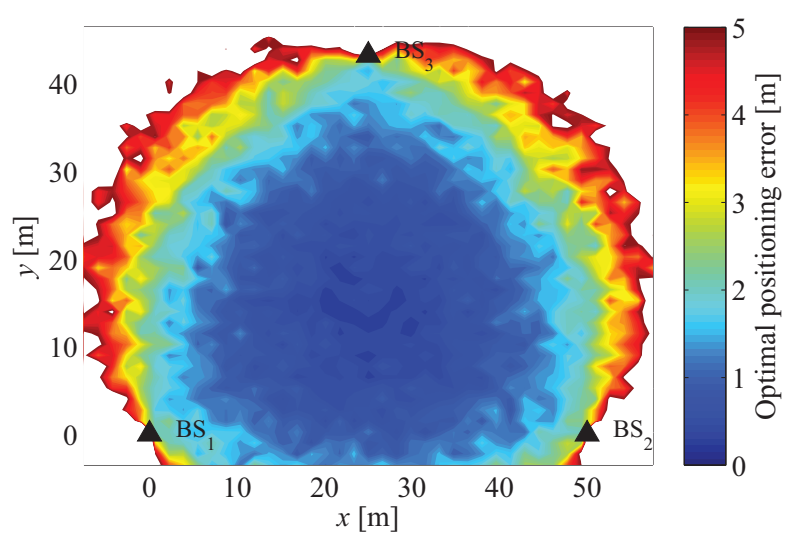

Figure 7. Optimal positioning error $\sigma_{\mathrm{opt}}=\sigma_{\mathrm{pos}}\left(\gamma_{\mathrm{opt}}\right)$ for the Diracrectangular waveform.

almost reaches the maximum of

$$
G_{\max }=1-\frac{\sigma_{\mathrm{pos}}(1)}{\sigma_{\mathrm{pos}}(0)}=1-\frac{1}{\sqrt{3}}=42,3 \%
$$

at the centroid of the triangular constellation It's in general lower than for free space path loss. The maximum gain is achieved when all the links' SNRs go to infinity. In this case the optimum waveform parameter $\gamma_{\mathrm{opt}} \rightarrow 1$ and we obtain (23) by applying the Cramér-Rao lower bound with (7), (8) and (16).

Since we use the minimum number of base stations to obtain a position estimate, one bad propagation scenario, i.e., NLOS propagation, already has a big impact on the positioning bound. For such a scenario, $\gamma_{\text {opt }} \rightarrow 0$ is more likely than $\gamma_{\mathrm{opt}} \rightarrow 1$ due to the high probability of having at least one low SNR value for the links to the BSs. In order to confirm the assertion that one bad link already affects the selection of the parameter $\gamma$ in a crucial manner, we conducted two more simulations, where we changed the geometry of the BSs in the first one and used the WINNER II channel model in the second one.

By adding a fourth BS, we increase the probability that we have at least three links with sufficiently high SNR values to estimate the position. This should directly influence the 


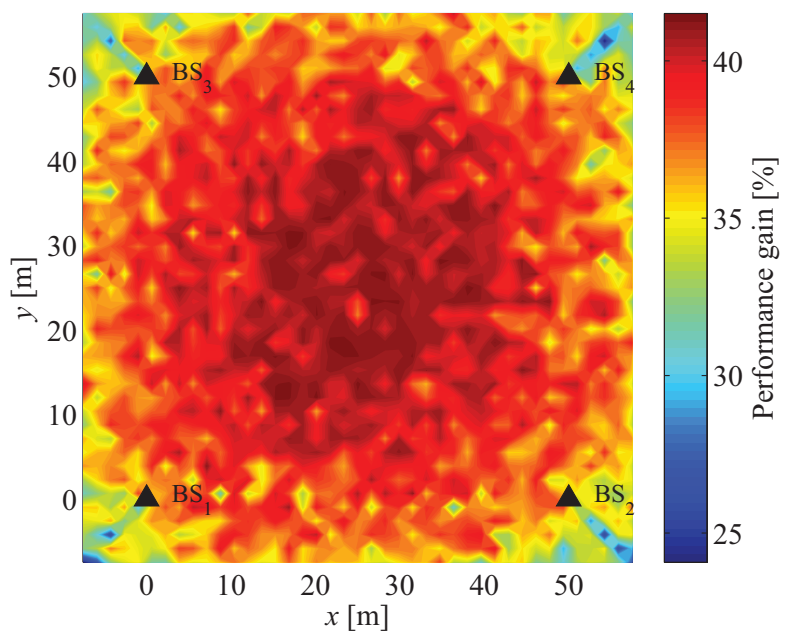

Figure 8. Performance gain $1-\sigma_{\mathrm{opt}} / \sigma_{\mathrm{pos}}(0)$ for the optimal waveform for a four BS scenario.

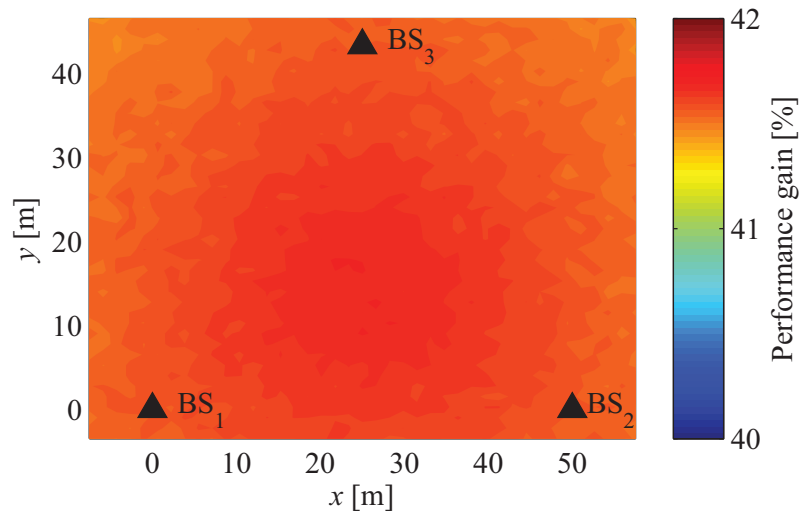

Figure 9. Performance gain $1-\sigma_{\mathrm{opt}} / \sigma_{\mathrm{pos}}(0)$ for the optimal waveform for purely LOS scenarios.

performance gain that can be achieved, since the probability of links with reasonable SNR values increases. The reasoning for such a scenario are future massive device-to-device links, where the probability of MVs in the vicinity, i.e., links with sufficiently high SNR values usable for position estimation, increases.

The results in Fig. 8 show that observing one additional link at the receiver helps in obtaining almost the optimal gain for the whole scenario. At virtually every position inside the rectangular area, the propagation conditions to three different BSs are most probable LOS conditions so that a high gain can be achieved as expected.

The last use case that we investigate uses the WINNER II channel model with the LOS probability equal to one, i.e., we always have LOS conditions with additional shadow fading. As it can be seen in Fig. 9, the propagation conditions are reasonable good within the triangular area between the BSs and the performance gain is hardly affected by shadow fading.

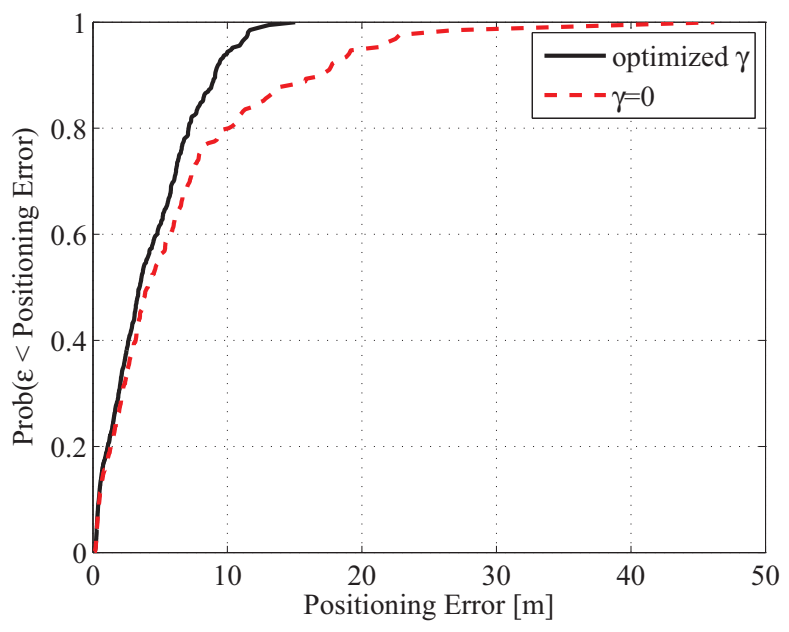

Figure 10. CDF of the positioning error for optimized and fixed $\gamma$.

Therefore we conclude that the NLOS propagation conditions have a crucial effect on the positioning performance. If the MV moves in a urban canyon with only three BSs available and at least one of them is blocked by a building, the performance gain is smaller and a rectangular waveform is more beneficial for ranging.

In Fig. 10, we show the cumulative density function of the positioning error for arbitrary positions in the triangular area and the normal WINNER II channel. The resulting curves show that the rectangular waveform performs worse than the optimized waveform. Until a cumulative probability function value of 0.5 both waveforms show a similar performance. At higher probabilities, the optimized waveform with $\gamma_{\mathrm{opt}}$ outperforms the waveform with $\gamma=0$.

\section{CONCLUSION}

We showed the impact on the selection of the waveform parameter using a realistic channel model for simulations. It becomes clear that the channel worsens the propagation conditions and therefore the spectrum waveform should be closer to a rectangular shape instead of concentrating all energy to the edges of the spectrum. This result, however, is only valid using a minimum of three base stations and under non-line-of-sight conditions. If there are more base stations available, the best links can be used for positioning and the optimized waveform diverges from a rectangular spectrum. If only line-of-sight conditions are prevalent, a power spectral density with energy concentrated at the edges is advantageous. With a flexible waveform it becomes possible to optimally adapt it to the propagation conditions in order to obtain the best positioning performance. The proposed network-based positioning with an adjustable waveform should complement the positioning service of global navigation satellite systems.

\section{ACKNOWLEDGMENT}

This work was partially supported by the EU project HIGHTS (High precision positioning for cooperative ITS applications) MG-3.5a-2014-636537 and the DLR project Dependable Navigation. 


\section{REFERENCES}

[1] "HIGHTS Deliverable D5.1: Cooperative Localization Algorithms and Hybrid Data Fusion Schemes," Jun. 2016. [Online]. Available: http://hights.eu/?page_id=699

[2] G. Fettweis and S. Alamouti, "5G: Personal mobile internet beyond what cellular did to telephony," IEEE Communications Magazine, vol. 52, no. 2, pp. 140-145, Feb. 2014.

[3] J. Andrews, S. Buzzi, W. Choi, S. Hanly, A. Lozano, A. Soong, and J. Zhang, "What will 5G be?" IEEE Journal on Selected Areas in Communications, vol. 32, no. 6, pp. 1065-1082, Jun. 2014.

[4] A. Osseiran, F. Boccardi, V. Braun, K. Kusume, P. Marsch, M. Maternia, O. Queseth, M. Schellmann, H. Schotten, H. Taoka, H. Tullberg, M. Uusitalo, B. Timus, and M. Fallgren, "Scenarios for 5G mobile and wireless communications: The vision of the METIS project," IEEE Communications Magazine, vol. 52, no. 5, pp. 26-35, May 2014.

[5] "5G White Paper," Dec. 2014, version 1.0, Final Executive Version. [Online]. Available: http://www.ngmn.org/uploads/media/ 141222_NGMN-Executive_Version_of_the_5G_White_Paper_v1_0.pdf
[6] B. Ben-Moshey, E. Elkinz, H. Levix, and A. Weissman, "Spreading code design for a MC-CDMA based GNSS pilot signal," in Proceedings of the Canadian Conference on Computational Geometry, Toronto, Canada, August 2011.

[7] C. Sturm and W. Wiesbeck, "Waveform design and signal processing aspects for fusion of wireless communications and radar sensing," Proceedings of the IEEE, vol. 99, no. 7, pp. 1236-1259, July 2011.

[8] "WINNER II Deliverable D1.1.2: WINNER II Channel Models," Sep 2007. [Online]. Available: http://www.ist-winner.org/deliverables.html

[9] A. Dammann, R. Raulefs, and S. Zhang, "On prospects of positioning in 5G," in Proceedings IEEE ICC 2015 - Workshop on 5G \& Beyond Enabling Technologies and Applications, London, UK, Jun. 2015.

[10] S. M. Kay, Fundamentals of Statistical Signal Processing - Estimation Theory. Prentice Hall, 1993.

[11] C. Musso and J.-P. Ovarlez, "Improvement of the Ziv-Zakai lower bound for time delay estimation," in 15th European Signal Processing Conference (EUSIPCO 2007), Poznan, Poland, Sep. 2007, pp. 960-964. 